\title{
Interactions between the enhanced recovery after surgery pathway and risk factors for lung infections after pulmonary malignancy operation
}

\author{
Xianfei Zhang' ${ }^{1 \#}$, Runsen Jin ${ }^{1 \#}$, Yuyan Zheng ${ }^{1 \#}$, Dingpei Han ${ }^{1}$, Kai Chen ${ }^{1}$, Jian $\mathrm{Li}^{2}$, Hecheng Li ${ }^{1}$ \\ ${ }^{1}$ Department of Thoracic Surgery, ${ }^{2}$ Department of Clinical Research Center, Ruijin Hospital, Shanghai Jiao Tong University School of Medicine, \\ Shanghai, China \\ Contributions: (I) Conception and design: X Zhang, R Jin; (II) Administrative support: H Li; (III) Provision of study materials or patients: X Zhang, \\ Y Zheng, D Han, K Chen, H Li; (IV) Collection and assembly of data: X Zhang, Y Zheng; (V) Data analysis and interpretation: X Zhang, J Li; (VI) \\ Manuscript writing: All authors; (VII) Final approval of manuscript: All authors. \\ "These authors contributed equally to this work. \\ Correspondence to: Hecheng Li, MD, PhD. Department of Thoracic Surgery, Ruijin Hospital, Shanghai Jiao Tong University School of Medicine, 197 \\ Ruijin 2nd Road, Shanghai 200025, China. Email: lihecheng2000@hotmail.com.
}

\begin{abstract}
Background: Lung infection is a common complication after thoracic surgery and can lead to severe consequences. Our study was designed to explore the risk factors for postoperative lung infections (POLI) following pulmonary malignancy operation and assess the protective effect of enhanced recovery after surgery (ERAS) and their potential interactive relationships.

Methods: A retrospective study included 1,768 patients who underwent surgery between 2013 and 2017 in Ruijin Hospital, Shanghai Jiaotong University School of Medicine was performed. Uni- and multivariate analyses were performed to identify risk factors. Andersson's model was applied to evaluate the additive interaction between these factors.

Results: Smoking [95\% confidence interval (CI): 1.178-2.198], preoperative heart disease (95\% CI: 1.448-4.091), and massive intraoperative blood loss (95\% CI: 1.568-3.674) were independent risk factors for postoperative lung infections (POLI), whereas ERAS implementation was protective (95\% CI: 0.249-0.441). Interaction analyses indicated that non-ERAS was reciprocally independent with smoking and surgical procedure. It had a synergistic interaction with heart disease [attributable proportion due to interaction (AP) $=0.540$ (95\% CI: 0.179-0.901), synergy index (S) =2.580 (95\% CI: 1.016-6.551)], and poor lung function [AP $=0.395$ (95\% CI: $0.016-0.775)]$, as well as a tendency of antagonistic interaction with blood loss.

Conclusions: Intraoperative blood loss, heart disease, and smoking are independent risk factors of POLI. ERAS implementation is a protective factor and is firstly verified to be more effective on reducing POLI in patients with heart diseases, poor lung function, and less intraoperative blood loss. We provide evidences to implement ERAS and a clue of the most optimal indications for ERAS.
\end{abstract}

Keywords: Postoperative lung infections (POLI); enhanced recovery after surgery (ERAS); interactive analysis

Submitted Mar 08, 2020. Accepted for publication Aug 05, 2020.

doi: $10.21037 /$ tlcr-20-401

View this article at: http://dx.doi.org/10.21037/tlcr-20-401

\section{Introduction}

Lung cancer is the most common cancer worldwide, as well as the cancer with the highest mortality rate $(1,2)$. Surgery is an essential component of comprehensive therapy for lung cancer but it also impairs patients' respiratory and circulatory function. A subset of patients who undergo surgery may suffer from postoperative complications, with lung infections accounting for the majority (3). The incidence of 
postoperative lung infections (POLI) ranges from 3.6\% to $20.3 \%$ (4-8). POLI can cause dyspnea, hypoxemia, and even systemic infections in the early postoperative period, leading to discomfort and increased hospitalization costs; it is also an important cause of early postoperative death and a lower long-term survival rate $(7,9,10)$.

Others have reported that risk factors for lung infections after pulmonary surgery mainly include age, lung function, smoking status, and respiratory and cardiovascular comorbidities $(7,8,11-13)$. On the other hand, enhanced recovery after surgery (ERAS) pathway which is a multimodal, multidisciplinary, and evidence-based approach to care surgical patients including perioperative nutritional support, fluid management, early extubation, and analgesia after surgery, has recently been shown to significantly decrease postoperative complications, length of hospital stay, and total cost (14-16). Controlling risk factors and implementing ERAS pathways are the two effective methods for reducing POLI. However, whether ERAS is effective regardless of the presence of risk factors is unknown.

Our clinical center, the thoracic surgery department of Ruijin Hospital, Shanghai Jiaotong University School of Medicine provides basic care for patients with lung cancer. Preoperative evaluation of patients' tolerance to the surgery and the possibility of complete resection of mass will be accomplished after admission. Anatomic pulmonary resection including lobectomy and segmentectomy with standard mediastinal lymph node dissection is carried out in the majority of suitable patients. An ERAS pathway, which was largely different from our traditional care, was generally implemented throughout the perioperative period in our department at the beginning of 2016.

The goals of our work were to identify risk factors for lung infections after pulmonary malignancy operation, to evaluate the potential protective effect of ERAS, and to perform interaction analyses between ERAS pathways and these risk factors to compare the effectiveness of ERAS between patients with and without risk.

We present the following article in accordance with the STROBE reporting checklist (available at http://dx.doi. org/10.21037/tlcr-20-401).

\section{Methods}

\section{Patient selection}

A retrospective study was made among patients who had developed pulmonary malignancy and underwent surgery between 2013 and 2017 in Ruijin Hospital, Shanghai Jiaotong University School of Medicine and a total of 1,908 patients were enrolled. Inclusion criteria were as follows: (I) patients who were diagnosed with pulmonary malignancy and underwent surgery to remove the lesion, (II) no elevated temperature or increased white blood cell (WBC) count before surgery, and (III) malignancies were verified by postoperative pathology. Accordingly, exclusion criteria were established as follows: (I) patients who couldn't meet the inclusion criteria, (II) patients with incomplete data, and (III) patients who refused to participate in the research.

\section{Predictors of POLI}

We collected patients' characteristics and preoperative status findings including sex, age, body mass index (BMI), tumor sites, forced expiratory volume in $1 \mathrm{~s} /$ forced vital capacity ratio (FEV1/FVC), smoke index, preoperative comorbidities (including diabetes, hypertension, heart diseases, respiratory system diseases), and whether they received neoadjuvant therapy and ERAS pathways. The characteristics of surgery were also collected including the surgical approach, range of lung excision, length of surgery, intraoperative blood loss, verified pathological outcomes, and whether they developed POLI.

The diagnosis of POLI mainly depends on symptoms, imaging tests, and microbiological examination $(4,6-8,12)$. However, a clear consensus has not been reached. We determined the diagnosis by combining symptoms with at least either imaging or microbiological tests to confirm a positive result. The standard is more rigorous than diagnosis based only on symptoms. Diagnostic criteria of POLI in our department include:

(I) Temperature $\geq 38^{\circ} \mathrm{C}$;

(II) $\mathrm{WBC}>10 \times 10^{9} / \mathrm{L}$ or $<4 \times 10^{9} / \mathrm{L}$;

(III) Newly developed cough and expectoration;

(IV) Newly developed moist rales or signs of pulmonary consolidation;

(V) Newly developed infiltration confirmed by computed tomography scan or chest $\mathrm{X}$-ray;

(VI) Microbiologic examination confirmation.

POLI was established if any one of items 1 to 4 and either item 5 or 6 existed.

\section{Statistical analysis}

SPSS Statistics 22.0 (IBM, Armonk, NY, USA) software were utilized to run statistical analyses. Differences were 
considered significant at $\mathrm{P}<0.05$. Univariate analyses were performed first. Categorical variables were analyzed by $\chi^{2}$ test or Fisher's exact test as appropriate. Student's $t$-tests were performed to analyze continuous variables that conformed to a normal distribution, and non-parametric testing was used for non-normal continuous variables. Those variables with significant differences were brought into multivariate analysis where binary stepwise regression analysis was applied to identify relevant factors associated with POLI. Continuous variables were transferred into categorical variables before regression analysis. The rules for transferring are listed below.

(I) Blood loss during surgery $\rightarrow<400$ or $\geq 400 \mathrm{~mL}$;

(II) Surgery time $\rightarrow<2$ or $\geq 2$ hours.

Significance were considered if the confidence interval (CI) of odds ratio (OR) did not include 0 . This step revealed which independent factors might affect the development of POLI.

To explore the latent additive interaction between protective and risk factors, Andersson's model was applied to calculate the relative excess risk due to interaction (RERI), attributable proportion due to interaction (AP), and synergy index $(\mathrm{S})$. The significance level $\alpha$ was set to 0.05. Differences were considered significant if the CI of RERI and AP did not include 0 and CI of $\mathrm{S}$ did not include 1, indicating an interaction between the two factors. Furthermore, synergy would be considered if at least one of three indexes conform to the inequalities: RERI $>0$, AP $>0$, or $\mathrm{S}>1$. Conversely, antagonism would be considered if $\mathrm{RERI}<0, \mathrm{AP}<0$ or $\mathrm{S}<1$. The two factors would be considered independent reciprocally if none of interactive indexes showed significant differences.

\section{Ethical statement}

All procedures performed in this study were in accordance with the Declaration of Helsinki (as revised in 2013) and approved by the Ethics Committee of the Ruijin Hospital, Shanghai Jiao Tong University School of Medicine (Registration number 2020166). Because of the retrospective nature of the research, the requirement for informed consent was waived.

\section{Results}

\section{Patient characteristics}

We analyzed a total of 1,768 patients who were diagnosed before surgery or were confirmed by postoperative pathological examination and $240(13.6 \%)$ of them developed POLI. Overall, 929 (52.5\%) patients were female and $839(47.5 \%)$ were male. The average age was 59.8 years old. The most common tumor location was the right upper lobe $(26.8 \%)$. Other basic health characteristics including BMI, lung function, preoperative comorbidities, and postoperative treatments are summarized in Table 1 .

\section{Risk and protective factors for POLI}

We first performed univariate analyses of preoperative patient characteristics (Table 2) and identified the following risk factors: male sex $(16.3 \%$ vs. $11.1 \%$, compared to female, $\mathrm{P}=0.001)$, smoking ( $20.0 \%$ vs. $11.7 \%$, compared to non-smoking, $\mathrm{P}<0.001)$, and heart disease $(23.5 \%$ vs. $13.0 \%$, compared to without heart disease, $\mathrm{P}=0.003$ ). Analyses of characteristics of surgery, postoperative care, and tumor nature (Table 3) showed that implementation of ERAS pathways $(23.8 \%$ to $8.7 \%, \mathrm{P}<0.001)$, open surgery ( $20.8 \%$ vs. $12.5 \%$, compared to minimally invasive, $\mathrm{P}=0.001$ ), extensive scale of surgery (such as dual lobectomy, $30.8 \%, \mathrm{P}<0.001)$, large cell carcinomas $(45.5 \%, \mathrm{P}<0.001)$, longer surgery time (165 vs. $140 \mathrm{~min}, \mathrm{P}<0.001)$, more intraoperative blood loss (120 vs. $100 \mathrm{~mL}, \mathrm{P}<0.001)$ were associated with a greater risk of POLI.

The variables that showed significance in the univariate analysis were brought into the multivariate analysis (Table 4). Stepwise binary logistic regression was applied so that the final variables entering the regression equation had statistically significant differences and could be considered as independent influencing factors. Smoking (OR 1.608; 95\% CI: 1.178-2.198), heart disease (OR 2.434; 95\% CI: 1.448-4.091), blood loss $\geq 400 \mathrm{~mL}$ (OR 2.400; 95\% CI: 1.568-3.674) and were determined as independent risk factors. Considering that surgery length is affected by many factors (the proficiency of surgeons and assistant nurses, the teaching curve for new surgeons, etc.), we believe that this variable is a confounding factor although it was significant.

Furthermore, implementation of ERAS (compared to noERAS implementation, OR 0.331; 95\% CI: 0.249-0.441) was confirmed as the only protective factor for POLI.

\section{Interaction between protective and risk factors}

We performed interaction analyses between ERAS and independent risk factors obtained in the multivariate analysis. Furthermore, risk factors reported in published 
Table 1 Descriptive characteristics of 1,768 patients

\begin{tabular}{|c|c|c|c|c|}
\hline Variable & $\mathrm{n}$ & $\%$ & Mean & SD \\
\hline \multicolumn{5}{|l|}{ Sex } \\
\hline Female & 929 & 52.5 & & \\
\hline Male & 839 & 47.5 & & \\
\hline Age $^{\star}$ & & & 59.810 & 11.435 \\
\hline $\mathrm{BMI}^{*}$ & & & 23.478 & 3.180 \\
\hline \multicolumn{5}{|l|}{ Smoke } \\
\hline No & 1,366 & 77.3 & & \\
\hline Yes & 401 & 22.7 & & \\
\hline \multicolumn{5}{|l|}{ Tumor site } \\
\hline Unknown & 31 & 1.8 & & \\
\hline Right upper lobe & 473 & 26.8 & & \\
\hline Right middle lobe & 108 & 6.1 & & \\
\hline Right lower lobe & 260 & 14.7 & & \\
\hline Left upper lobe & 335 & 18.9 & & \\
\hline Left lower lobe & 217 & 12.3 & & \\
\hline Multiple & 344 & 19.5 & & \\
\hline \multicolumn{5}{|l|}{ FEV1/FVC } \\
\hline$\geq 0.7$ & 1,604 & 90.7 & & \\
\hline$<0.7$ & 164 & 9.3 & & \\
\hline \multicolumn{5}{|l|}{ Diabetes } \\
\hline No & 1,593 & 90.1 & & \\
\hline Yes & 175 & 9.9 & & \\
\hline \multicolumn{5}{|l|}{ Hypertension } \\
\hline No & 1,219 & 68.9 & & \\
\hline Yes & 549 & 31.1 & & \\
\hline \multicolumn{5}{|l|}{ Heart diseases } \\
\hline No & 1,670 & 94.5 & & \\
\hline Yes & 98 & 5.5 & & \\
\hline \multicolumn{5}{|l|}{ Respiratory diseases } \\
\hline No & 1,730 & 97.9 & & \\
\hline Yes & 38 & 2.1 & & \\
\hline \multicolumn{5}{|c|}{ Neoadjuvant chemotherapy } \\
\hline No & 1,743 & 98.6 & & \\
\hline Yes & 25 & 1.4 & & \\
\hline
\end{tabular}

*, continuous variables are presented as means and SDs. articles besides surgery approach and lung function were also analyzed. No-ERAS was considered as a risk factor for the convenience of calculation and result explanation. The incidence rates for different conditions are shown in Figure 1, and the results of the interaction analysis are shown in Table 5 and Figure 2.

ERAS was reciprocally independent with smoking as none of the three interactive indexes showed significant differences [RERI $=0.195$ (95\% CI: -2.025 to 2.416 ), AP $=0.037$ (95\% CI: -0.375 to 0.450 ), S=1.048 (95\% CI: 0.616 to 1.782)]. In other words, ERAS had the same effect in reducing POLI for smoking and non-smoking patients. ERAS was also reciprocally independent with surgical procedure for the same reason [RERI $=-0.170$ (95\% CI: -2.400 to 2.061 ), $\mathrm{AP}=-0.040$ (95\% CI: -0.582 to 0.481 ), $\mathrm{S}=0.950$ (95\% CI: 0.481 to 1.874$)]$. Therefore, ERAS is effective for patients whether they undergo open or minimally invasive surgery.

ERAS was more effective in reducing the risk of POLI in patients with heart disease, as two of three interactive indexes showed significant differences [RERI $=4.541(95 \%$ OR: -1.537 to 10.620$), \mathrm{AP}=0.540$ (95\% OR: $0.179-0.901$ ), $\mathrm{S}=2.580$ (95\% OR: 1.016-6.551)]. Moreover, patients with poor lung function can benefit more from ERAS pathways, as indicated by the significant difference for AP [RERI $=2.079$ (95\% OR: -0.961 to 5.120$), \mathrm{AP}=0.395$ (95\% OR: 0.016-0.775), $\mathrm{S}=1.954$ (95\% OR: 0.863-4.424)].

None of the three indexes were significantly different between ERAS and intraoperative blood loss [RERI $=-1.584$ (95\% OR: -6.295 to 3.126$), \mathrm{AP}=-0.238$ (95\% OR: -1.050 to 0.574 ), $\mathrm{S}=0.781$ (95\% OR: -0.364 to 1.677 )]. Even so, a tendency was observed in that patients with minimal intraoperative blood loss might benefit more from ERAS.

\section{Discussion}

POLI is one of the most common complications after pulmonary surgery (3). Patients with POLI suffer from cough, excessive phlegm, fever and even respiratory failure. Encouraging the patients to expectorate and applying antibiotics are two main methods to treat POLI. On the other hand, finding and controlling risk factors is helpful to reduce the morbidity of POLI. Others have reported that risk factors for POLI mainly include age, lung function, smoking status, and respiratory and cardiovascular comorbidities $(7,8,11-13)$. Our study explored and found 
Table 2 Univariate analysis of preoperative patient characteristics

\begin{tabular}{|c|c|c|c|c|c|c|}
\hline Variable & $\mathrm{n}$ & \multicolumn{2}{|c|}{ Lung infections, n (\%) } & $\chi^{2}$ value & $T$ value & $P$ value \\
\hline \multicolumn{7}{|l|}{ Sex } \\
\hline Female & 929 & 826 & $103(11.1)$ & & & \\
\hline Male & 839 & 702 & 137 (16.3) & 10.325 & & 0.001 \\
\hline $\mathrm{BMI}^{*}$ & & $23.43 \pm 3.098$ & $23.79 \pm 3.651$ & & -1.444 & 0.150 \\
\hline \multicolumn{7}{|l|}{ Smoke } \\
\hline No & 1,366 & 1,206 & $160(11.7)$ & & & \\
\hline Yes & 401 & 321 & $80(20.0)$ & 17.920 & & $<0.001$ \\
\hline Right upper lobe & 473 & 404 & $69(14.6)$ & & & \\
\hline Right middle lobe & 108 & 99 & $9(8.3)$ & & & \\
\hline Right lower lobe & 260 & 228 & $32(12.3)$ & & & \\
\hline Left upper lobe & 335 & 287 & $48(14.3)$ & & & \\
\hline Left lower lobe & 217 & 180 & $37(17.1)$ & & & \\
\hline Multiple & 344 & 301 & $43(12.5)$ & 7.375 & & 0.288 \\
\hline \multicolumn{7}{|l|}{ FEV1/FVC } \\
\hline$\geq 0.7$ & 1,604 & 1,393 & $211(13.2)$ & & & \\
\hline No & 1,219 & 1,052 & $167(13.7)$ & & & \\
\hline Yes & 549 & 476 & 73 (13.3) & 0.052 & & 0.819 \\
\hline \multicolumn{7}{|l|}{ Heart diseases } \\
\hline No & 1,670 & 1,453 & $217(13.0)$ & & & \\
\hline Yes & 98 & 75 & $23(23.5)$ & 8.658 & & 0.003 \\
\hline \multicolumn{7}{|l|}{ Respiratory diseases } \\
\hline No & 1,730 & 1,495 & $235(13.6)$ & & & \\
\hline Yes & 38 & 33 & $5(13.2)$ & 0.006 & & 0.940 \\
\hline \multicolumn{7}{|c|}{ Neoadjuvant chemotherapy } \\
\hline No & 1,743 & 1,510 & $233(13.4)$ & & & \\
\hline Yes & 25 & 18 & $7(28.0)$ & 3.626 & & 0.068 \\
\hline
\end{tabular}

${ }^{*}$, variates including age (years), BMI $\left(\mathrm{kg} / \mathrm{m}^{2}\right)$ conformed to normal distribution and were analyzed by $t$-tests; the means, SDs, and T and $\mathrm{P}$ values are given in the table. Categorical data were analyzed by $\chi^{2}$ tests, the $\chi^{2}$ and $P$ values are given in the table. 
Table 3 Univariate analysis of surgery characteristics, postoperative care, and tumor nature

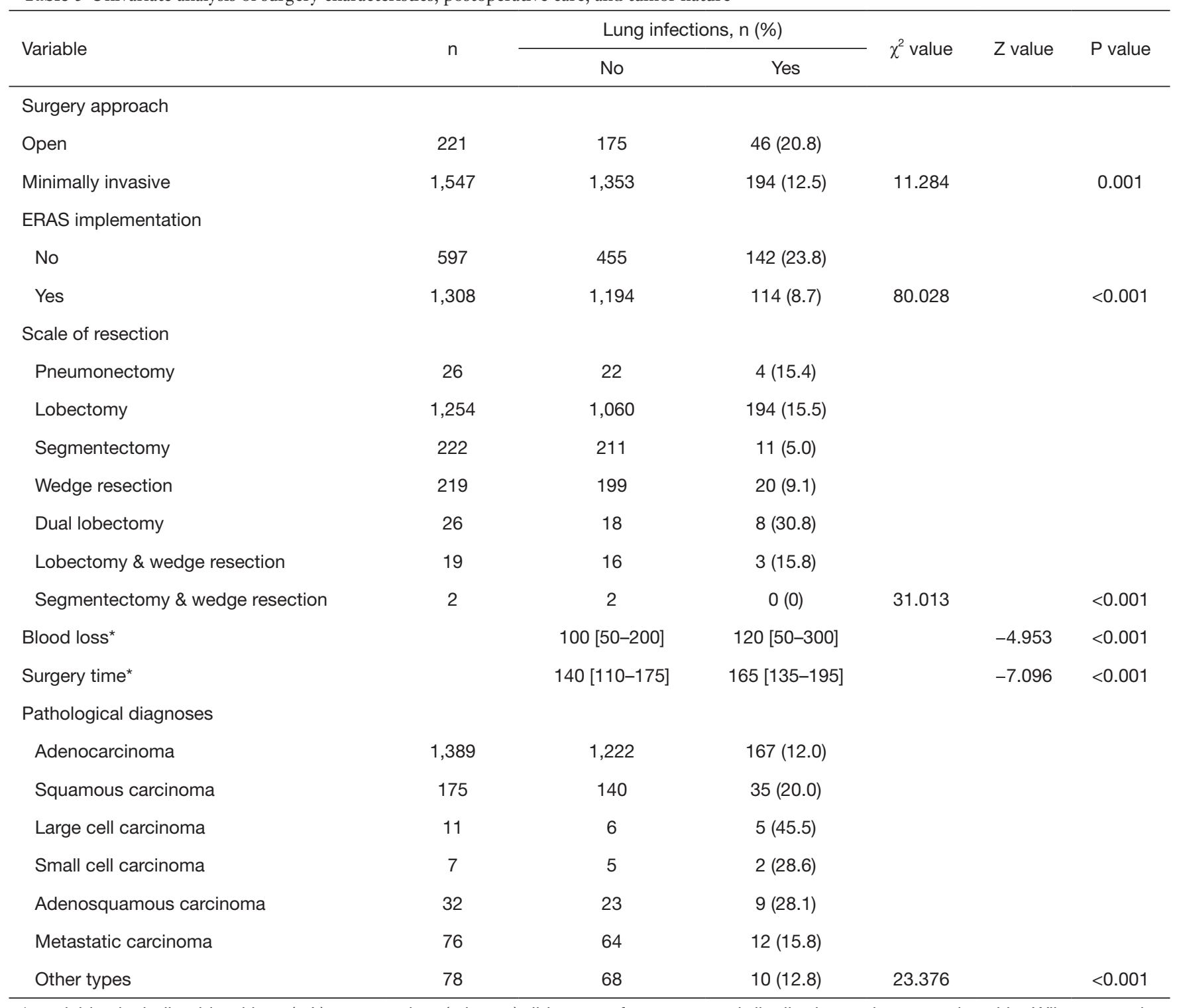

*, variables including blood loss $(\mathrm{mL})$, surgery time (minutes) did not conform to normal distribution and were analyzed by Wilcoxon ranksum test; the median, first and third quartiles, $Z$ values, and $P$ values are given in the table. Categorical data were analyzed by $\chi^{2}$ tests, the $\chi^{2}$ and $P$ values are given in the table.

that smoking, heart disease, massive blood loss during surgery were independent risk factors for POLI, which is consistent with other reports. However, other factors such as age, $\mathrm{BMI}, \mathrm{FEV}_{1} / \mathrm{FVC}$ and surgical approach were nonsignificant according to our findings.

Smoking considerably impairs lung function. Increased secretions and airway spasms caused by smoking lead to airway stenosis. As a result, the incidence of POLI in smoking patients is significantly increased $(17,18)$.
Patients with preoperative heart disease also have an increased likelihood of POLI, possibly due to decreased pulmonary circulatory capacity after lung surgery, which leads to increased pressure in the pulmonary circulation and increased exudation. Nojiri et al. demonstrated that preoperative elevated brain natriuretic peptide levels are significantly associated with postoperative cardiopulmonary complications, and low-dose atrial natriuretic peptides administration can reduce postoperative cardiopulmonary 
Table 4 Risk and protective factors determined by stepwise binary logistic regression

\begin{tabular}{|c|c|c|c|c|c|c|c|}
\hline Risk factor & B & Standard deviation & Wald & $\mathrm{P}$ & OR & \multicolumn{2}{|c|}{$95 \% \mathrm{Cl}$} \\
\hline Smoke & 0.475 & 0.160 & 8.845 & 0.003 & 1.608 & 1.176 & 2.198 \\
\hline Heart disease & 0.889 & 0.265 & 11.263 & 0.001 & 2.434 & 1.448 & 4.091 \\
\hline ERAS & -1.106 & 0.146 & 57.442 & $<0.001$ & 0.331 & 0.249 & 0.441 \\
\hline Blood loss & 0.876 & 0.217 & 16.263 & $<0.001$ & 2.400 & 1.568 & 3.674 \\
\hline Constant & -3.170 & 0.363 & 76.133 & $<0.001$ & 0.042 & - & - \\
\hline
\end{tabular}

$\mathrm{Cl}$, confidence interval; OR, odds ratio.

complications $(19,20)$. These evidences support the hypothesis that heart disease can cause perioperative cardiac dysfunction and induce POLI. In addition, a moderate to large volume of blood loss during surgery also precipitated POLI. Ogawa et al. pointed out that the degree of intraoperative blood loss is associated with the occurrence of postoperative complications in the elderly (21). Massive blood loss can cause insufficient blood volume, contraction of the pulmonary vascular bed, and decreased blood perfusion of the lungs, thereby reducing local anti-infective ability. Considerable intraoperative blood loss can increase the need for blood transfusion, which may increase the risk of postoperative infection $(22,23)$. As we found that smoking, heart disease, massive blood loss during surgery were independent risk factors for POLI, to quit smoking, to control heart diseases before surgery and for surgeons to control the intraoperative blood loss are theoretically effective to reduce lung infection after pulmonary malignancy operation.

Furthermore, we verified the role of ERAS in reducing POLI by identifying ERAS implementation as a protective factor. Our clinical care center generally implemented ERAS at the beginning of 2016 and the approaches include:

(I) Preoperative education and smoking cessation;

(II) Pre- and postoperative lung function evaluation and breathing training;

(III) Pre- and postoperative nutrition evaluation and support;

(IV) Postoperative pain evaluation and analgesia;

(V) Physical fitness assessment and early postoperative activities;

(VI) Drainage assessment and early postoperative extubation.

The largest difference between ERAS pathway and out traditional care is that we invite rehabilitation therapists to evaluate, instruct and supervise patients throughout the perioperative period to assure these approaches were carried out effectively and scientifically, rather than a simple oral instruction to patients as we used to do.

As a result of ERAS pathways, preoperative education and smoking cessation decrease lung secretions and reduce bronchospasm after surgery. Pre- and postoperative breathing training are beneficial to restore lung volume and function. Postoperative analgesia, nutrition support, and early activities and extubation help patients strengthen their respiratory movement, which can enhance gas exchange and facilitate effective coughing. All these methods reduce secretions in the respiratory tract and increase ventilatory volume, thus reducing the incidence of POLI.

The effect of ERAS may vary depending on patient status, but few studies have assessed this. To detect the differential effect of ERAS in the presence of risk factors, we innovatively performed an additive interaction analysis between ERAS and other risk factors. Although surgical approach and lung function were not independent risk factors according to our findings, they were also analyzed as they have been described in other published works $(6,12,24)$.

The final analysis showed that ERAS may have same effect regardless of smoking status. Secretion accumulation is a main cause of POLI and is severe in smokers, but it also exists in non-smokers because of anesthesia. ERAS reduces postoperative pulmonary secretion accumulation and therefore similarly decreases POLI in both non-smokers and smokers. The same effect was found between patients with minimally invasive surgery and open surgery. This may be due to the fact that open surgery is often performed for patients with complicated conditions. The high incidence of 


$$
\text { A }
$$

\section{A}

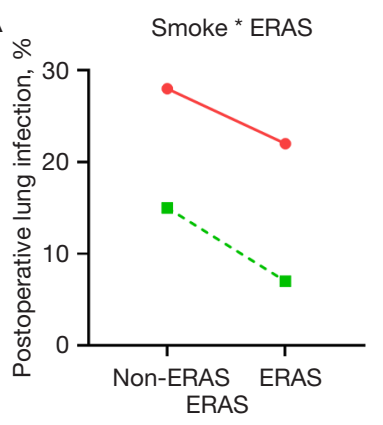

C

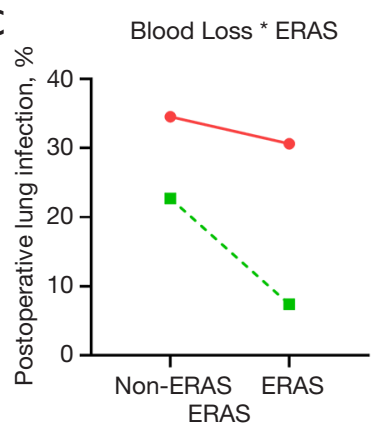

E

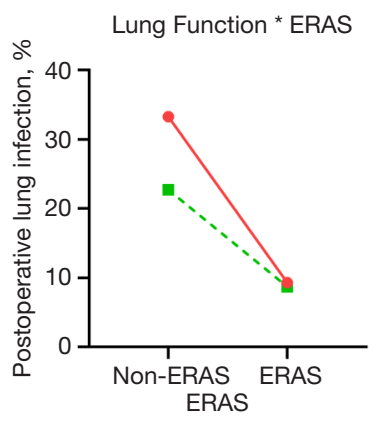

B

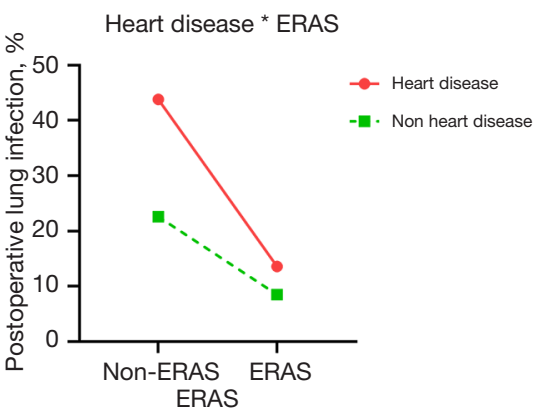

- Massive

-1.r. Slight

- Abnormal

-n. Normal

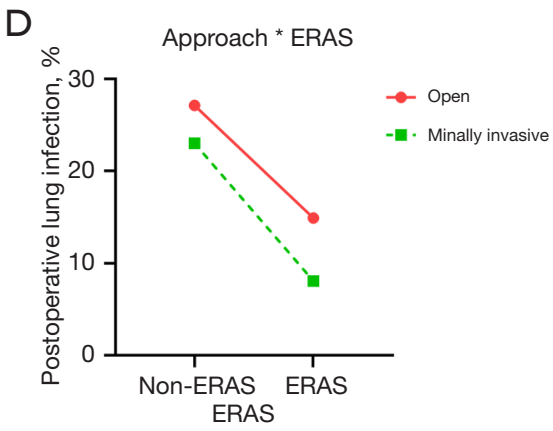

Figure 1 The effects of different risk factors on POLI rate. (A) ERAS has similar effects in smokers and nonsmokers. (B) ERAS is more effective in patients with heart disease. (C) ERAS is less effective in patients with minimal blood loss. (D) ERAS has similar effects regardless of surgery approach. (E) ERAS is more effective in patients with poor lung function. POLI, postoperative lung infections; ERAS, enhanced recovery after surgery.

lung infections is more determined by the condition itself (e.g., need for pneumonectomy) rather than postoperative care.

On the other hand, ERAS is more effective in patients with heart diseases and those with poor lung function. Patients with heart diseases or poor lung function may spend a long time in the bed and had less activity after surgery, which may aggravate lung congestion and contribute to the occurrence of POLI. Once ERAS was implemented, early postoperative activities and effective cough increased, cardiac and lung function enhanced, thereby congestion alleviated and secretion decreased, which reduce the rate of POLI.

Moreover, a tendency was observed that ERAS is probably more effective in patients with lower intraoperative blood loss. In patients with massive blood loss, the pulmonary vascular bed is contracted, so pulmonary congestion is milder and there is less lung secretion. Thus, the effect of ERAS to reduce POLI by alleviating pulmonary congestion and decrease lung secretion was weak 


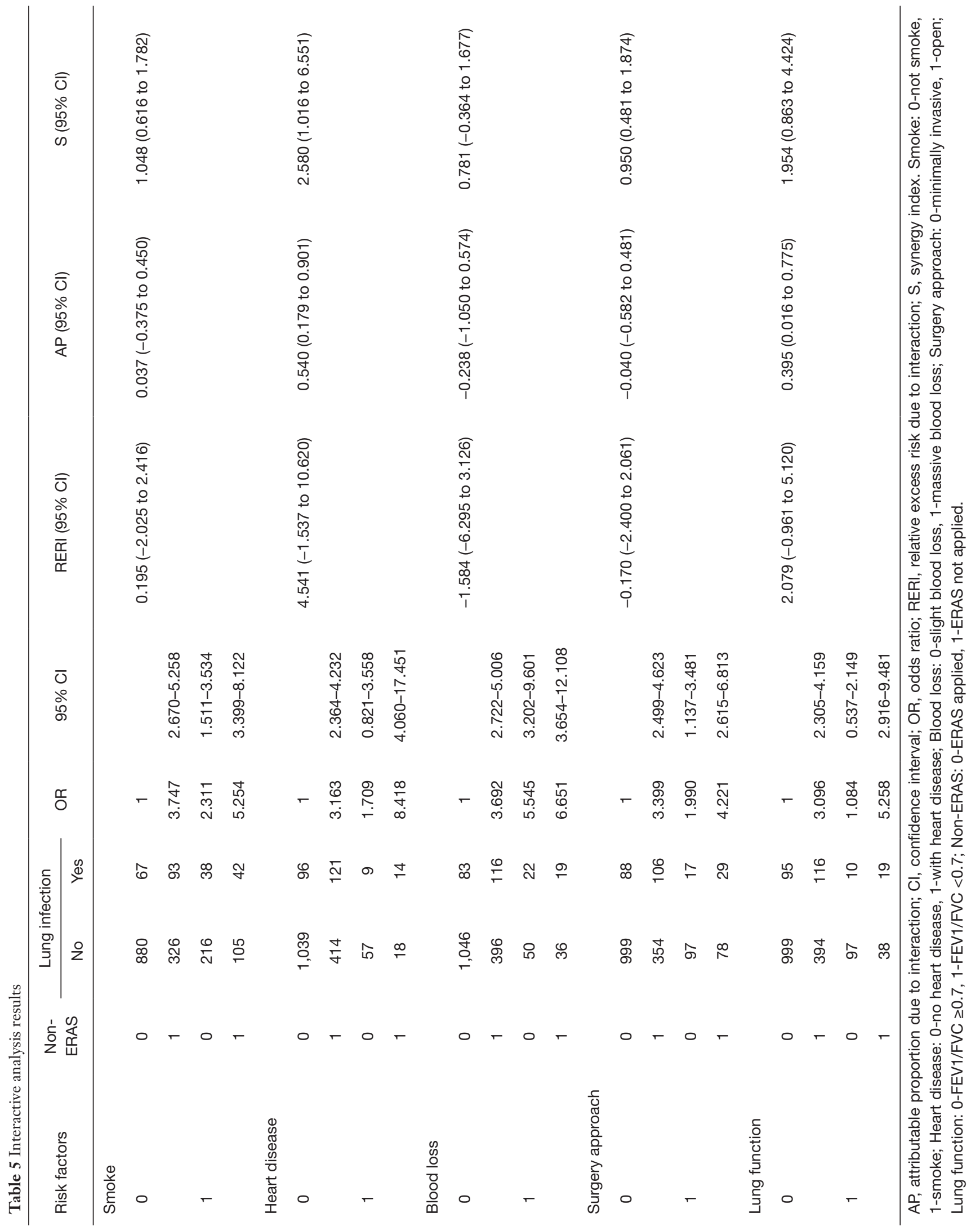




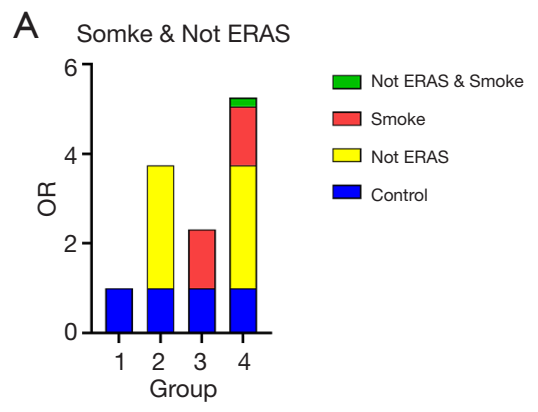

B Heart disease \& Not ERAS
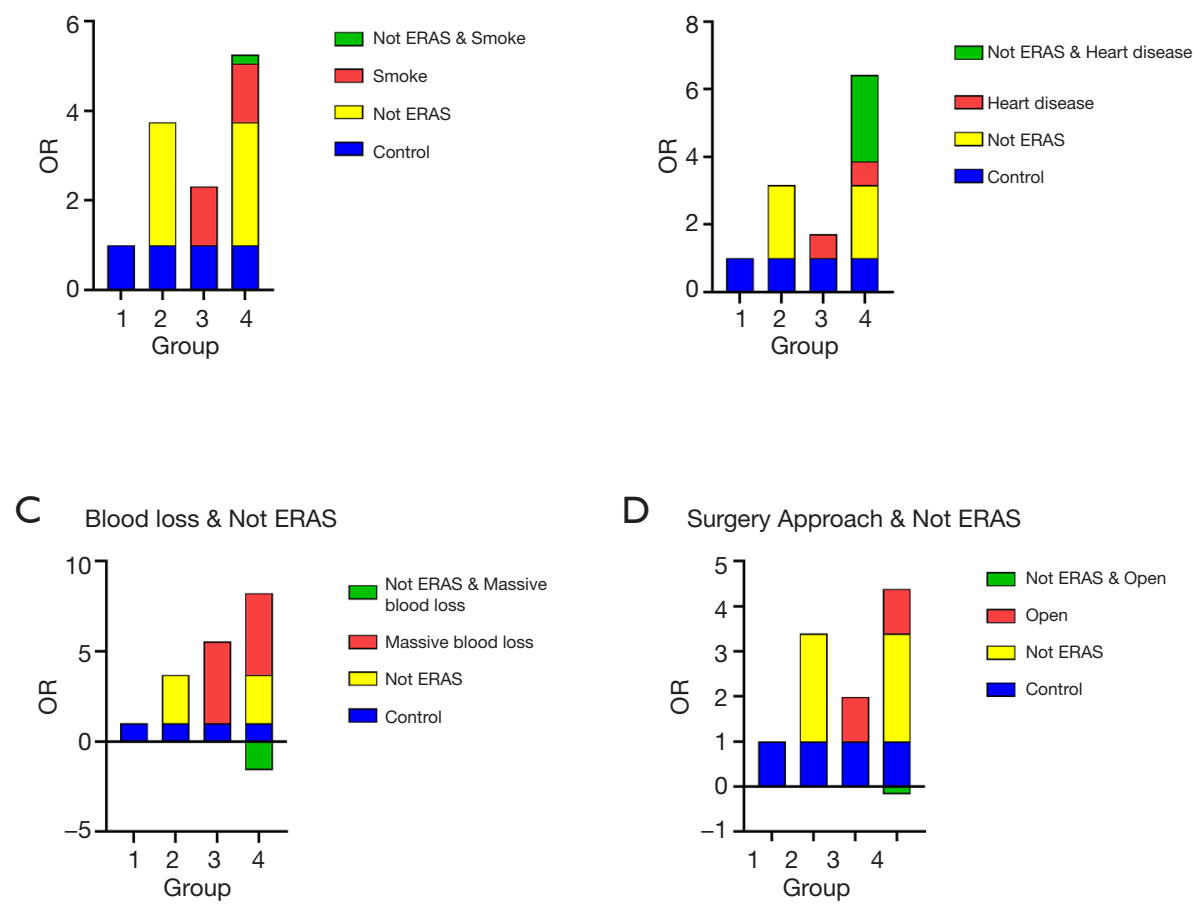

D Surgery Approach \& Not ERAS
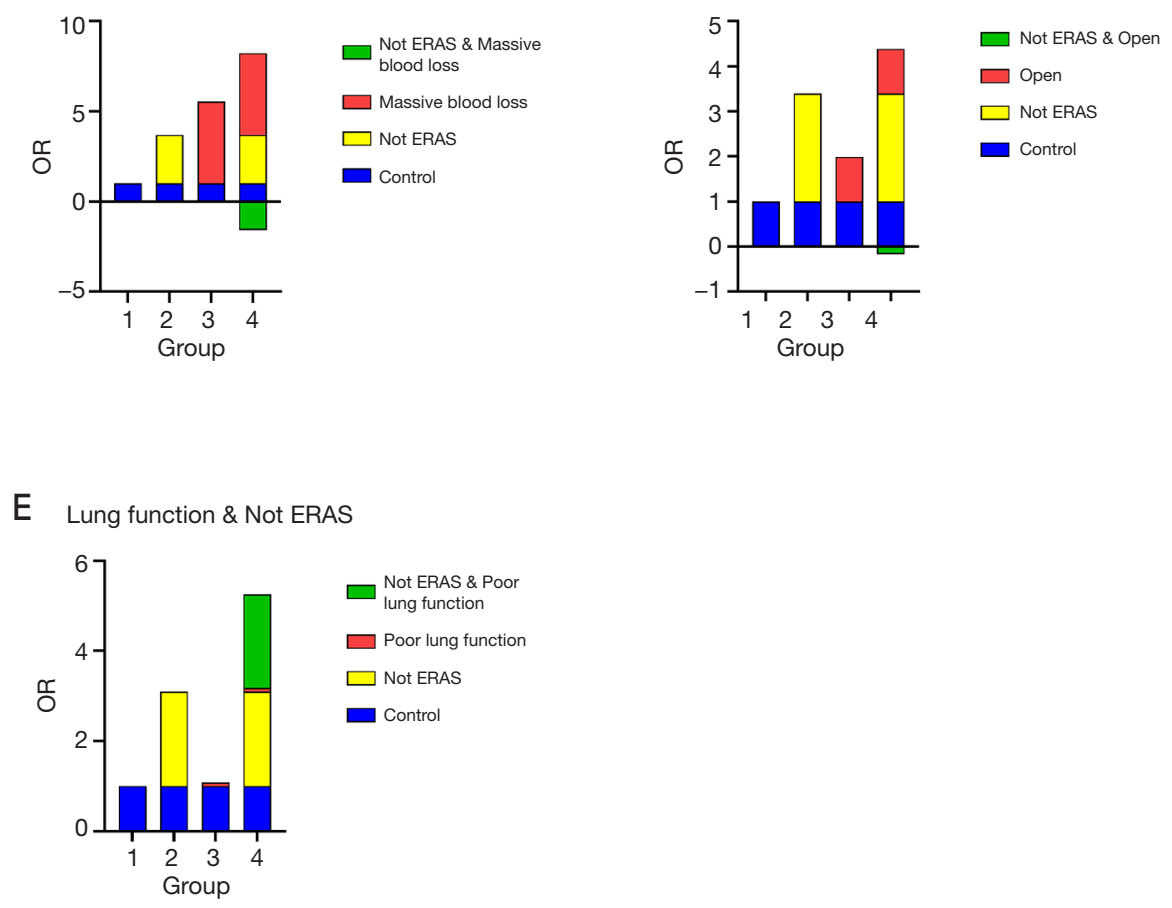

Figure 2 The visualized results of interactive analysis. (A) ERAS is reciprocally independent with smoking. (B) Not-ERAS has a synergistic effect with heart disease. (C) Not-ERAS has an antagonistic effect with massive intraoperative blood loss. (D) Not-ERAS is reciprocally independent of surgical approach. (E) Not-ERAS has a synergistic effect with poor lung function. ERAS, enhanced recovery after surgery.

in patients with more blood loss.

In conclusion, we found that preoperative smoking, massive intraoperative blood loss, and preoperative heart disease are independent risk factors for POLI, and the use of ERAS is a protective factor. Controlling risk factors and extensively implementing ERAS are important measures to reduce infections in patients undergoing surgery for lung malignancies. We innovatively performed an interactive analysis between ERAS and other risk factors to explore the protective effect of ERAS in different settings. The results are important to guide the selection patients who are more likely to benefit from ERAS. Ultimately, we for the first time found that ERAS is more effective in patients with heart disease and poor lung function, so it is more essential to emphasize the use of ERAS in these individuals. In addition, ERAS may achieve better outcomes in patients with less intraoperative blood loss, but further investigation is required. 


\section{Study limitations}

A rigorous standard of POLI diagnosis was adopted in our study to increase specificity. Consequently, some mild lung infections might have been ignored and the incidence might be underestimated. This is likely also we did not find significant associations for some risk factors described by other researchers. Moreover, it was a retrospective, single center study. Due to the lack of randomization, many influencing factors could not be controlled. Further randomized clinical trials are needed to eliminate bias and confirm the interaction between ERAS and specific risk factors.

\section{Acknowledgments}

Funding: Young Scientists Fund from National Natural Science Foundation of China (NSFC, 8170110124), Shanghai Municipal Education Commission-Gaofeng Clinical Medicine Grant Support (20172005), Shanghai Municipal Commission of Health and Family Planning Outstanding Academic Leaders Training Program (2017BR055) and Young Scientists Fund (20164Y0253).

\section{Footnote}

Reporting Checklist: The authors have completed the STROBE reporting checklist. Available at http://dx. doi. org/10. 21037/tlcr-20-401

Data Sharing Statement: Available at http://dx. doi. org/10. 21037/tlcr-20-401

Peer Review File: Available at http://dx. doi. org/10.21037/ tlcr-20-401

Conflicts of Interest: All authors have completed the ICMJE uniform disclosure form (available at http://dx. doi. org/10. 21037/tlcr-20-401). The authors have no conflicts of interest to declare.

Ethical Statement: The authors are accountable for all aspects of the work in ensuring that questions related to the accuracy or integrity of any part of the work are appropriately investigated and resolved. All procedures performed in this study were in accordance with the Declaration of Helsinki (as revised in 2013) and approved by the Ethics Committee of the Ruijin Hospital, Shanghai
Jiao Tong University School of Medicine (Registration number 2020166). Because of the retrospective nature of the research, the requirement for informed consent was waived.

Open Access Statement: This is an Open Access article distributed in accordance with the Creative Commons Attribution-NonCommercial-NoDerivs 4.0 International License (CC BY-NC-ND 4.0), which permits the noncommercial replication and distribution of the article with the strict proviso that no changes or edits are made and the original work is properly cited (including links to both the formal publication through the relevant DOI and the license). See: https://creativecommons.org/licenses/by-nc-nd/4.0/.

\section{References}

1. Chen $W$, Zheng R, Baade PD, et al. Cancer statistics in China, 2015. CA Cancer J Clin 2016;66:115-32.

2. Siegel RL, Miller KD, Jemal A. Cancer statistics, 2018. CA Cancer J Clin 2018;68:7-30.

3. Schussler O, Alifano M, Dermine H, et al. Postoperative pneumonia after major lung resection. Am J Respir Crit Care Med 2006;173:1161-9.

4. Diaz-Ravetllat V, Ferrer M, Gimferrer-Garolera JM, et al. Risk factors of postoperative nosocomial pneumonia after resection of bronchogenic carcinoma. Respir Med 2012;106:1463-71.

5. Dżeljilji A, Karuś K, Kierach A, et al. Efficacy and safety of pleurectomy and wedge resection versus simple pleurectomy in patients with primary spontaneous pneumothorax. J Thorac Dis 2019;11:5502-8.

6. Shiono S, Yoshida J, Nishimura M, et al. Risk factors of postoperative respiratory infections in lung cancer surgery. J Thorac Oncol 2007;2:34-8.

7. Simonsen DF, Sogaard M, Bozi I, et al. Risk factors for postoperative pneumonia after lung cancer surgery and impact of pneumonia on survival. Respir Med 2015;109:1340-6.

8. Yang R, Wu Y, Yao L, et al. Risk factors of postoperative pulmonary complications after minimally invasive anatomic resection for lung cancer. Ther Clin Risk Manag 2019;15:223-31.

9. Andalib A, Ramana-Kumar AV, Bartlett G, et al. Influence of postoperative infectious complications on long-term survival of lung cancer patients: a population-based cohort study. J Thorac Oncol 2013;8:554-61.

10. Lugg ST, Agostini PJ, Tikka T, et al. Long-term impact of 
developing a postoperative pulmonary complication after lung surgery. Thorax 2016;71:171-6.

11. Agostini P, Cieslik H, Rathinam S, et al. Postoperative pulmonary complications following thoracic surgery: are there any modifiable risk factors? Thorax 2010;65:815-8.

12. Lee JY, Jin SM, Lee CH, et al. Risk factors of postoperative pneumonia after lung cancer surgery. J Korean Med Sci 2011;26:979-84.

13. Bédat B, Abdelnour-Berchtold E, Krueger T, et al. Impact of complex segmentectomies by video-assisted thoracic surgery on peri-operative outcomes. J Thorac Dis 2019;11:4109-18.

14. Fiore JF Jr, Bejjani J, Conrad K, et al. Systematic review of the influence of enhanced recovery pathways in elective lung resection. J Thorac Cardiovasc Surg 2016;151:708-15.e706.

15. Rogers LJ, Bleetman D, Messenger DE, et al. The impact of enhanced recovery after surgery (ERAS) protocol compliance on morbidity from resection for primary lung cancer. J Thorac Cardiovasc Surg 2018;155:1843-52.

16. Elkhayat H, Gonzalez-Rivas D. ERAS in VATS-do we really need to follow the trend? Transl Lung Cancer Res 2019;8:S451-3.

17. Lugg ST, Tikka T, Agostini PJ, et al. Smoking and timing of cessation on postoperative pulmonary complications after curative-intent lung cancer surgery. J Cardiothorac Surg 2017;12:52.

18. Allinson JP, Hardy R, Donaldson GC, et al. Combined

Cite this article as: Zhang X, Jin R, Zheng Y, Han D, Chen K, $\mathrm{Li}$ J, Li H. Interactions between the enhanced recovery after surgery pathway and risk factors for lung infections after pulmonary malignancy operation. Transl Lung Cancer Res 2020;9(5):1831-1842. doi: 10.21037/tlcr-20-401
Impact of Smoking and Early-Life Exposures on Adult Lung Function Trajectories. Am J Respir Crit Care Med 2017;196:1021-30.

19. Nojiri T, Inoue M, Shintani Y, et al. B-type natriuretic peptide-guided risk assessment for postoperative complications in lung cancer surgery. World J Surg 2015;39:1092-8.

20. Nojiri T, Inoue M, Yamamoto K, et al. Effects of lowdose human atrial natriuretic peptide for preventing post-operative cardiopulmonary complications in elderly patients undergoing pulmonary resection for lung cancer. Eur J Cardiothorac Surg 2012;41:1330-4.

21. Ogawa F, Wang G, Matsui Y, et al. Risk factors for postoperative complications in the elderly with lung cancer. Asian Cardiovasc Thorac Ann 2013;21:313-8.

22. Horvath KA, Acker MA, Chang H, et al. Blood transfusion and infection after cardiac surgery. Ann Thorac Surg 2013;95:2194-201.

23. Xiao H, Quan H, Pan S, et al. Impact of peri-operative blood transfusion on post-operative infections after radical gastrectomy for gastric cancer: a propensity score matching analysis focusing on the timing, amount of transfusion and role of leukocyte depletion. J Cancer Res Clin Oncol 2018;144:1143-54.

24. Kim ES, Kim Y'T, Kang CH, et al. Prevalence of and risk factors for postoperative pulmonary complications after lung cancer surgery in patients with early-stage COPD. Int J Chron Obstruct Pulmon Dis 2016;11:1317-26. 